\title{
A Multi-Objective Approach for a Multihoming Load Balancing Scheme in WHN
}

\author{
C. Lozano-Garzon, M. Molina, Y. Donoso
}

\author{
Carlos Lozano-Garzon* \\ Universidad de los Andes, Bogotá, Colombia, South America \\ \& Universitat de Girona, Girona, Spain. \\ *Corresponding author: calozanog@ieee.org

\section{Miguel Molina, Yezid Donoso} \\ Universidad de los Andes \\ Bogotá, Colombia, South America \\ mf.molina35@uniandes.edu.co,ydonoso@uniandes.edu.co
}

\begin{abstract}
The telco operators face up to challenges related to the need of ensuring a quality of service to the user in a planning, maintenance and resource allocation in their complex networks. These challenges are directly related with the need to ensure an user's service with a good level of quality in a highly dynamic environment in terms of changes in the radio access technologies, growth in the number of mobile users, technical requirements of the new services and applications, and the possibility to connect to different networks at the same time, among others. In this paper, we address the problem of the user's service allocation into the different feasible networks in order to reduce the network overloading. We present a multihoming load balancing scheme that allows the re-allocation of services according to their QoS requirements and the availability of network resources. We propose a multi-objective optimization model of this problem together with an evolutionary algorithm to solve it. Through simulation in different scenarios, we show that our algorithm is efficient, sensitive, scalable and provides optimal solutions.

Keywords: Heterogeneous Networks, load Balancing, multihoming, multi-objective optimization, multi-objective evolutionary algorithms, vertical handover.
\end{abstract}

\section{Introduction}

Given the continuous advances in network technologies, the growth in the number of mobile users and the increasing demand of the new services and applications, the mobile network operator are confronted with multiple challenges in the planning, maintenance and operation of their complex infrastructure. In many cases, these networks are composed by multiple radio access technologies, which allows to the user the access to different services by using simultaneously one or more their network interfaces.

In some cases during the network operation, it is possible that some radio access channels could be overloaded because of a traffic growth caused by the increased of the number of user's services connected to these channels. Therefore, the mobile operators need some mechanisms that allows a balanced distribution of the traffic load over the available networks. Note that this process could involve could involve the reallocation of some users. The main goal of this mechanism is the optimal use of the available network resources whilst the technical requirements needs of each service are guaranteed.

From the viewpoint of the mobile network operators, one of the most appropriate ways to achieve this goal is that the deployed infrastructure must be capable to perform the user's reallocation for one network to another, this process is called Vertical Handover (VHO). Due to standards such as IEEE 802.21 [1] only provide the framework for VHO, the decision making 
algorithm that allow establish the best connection for each user is an open challenge [2]. In this algorithms, the re-allocation must be based on different metrics obtained from the mobile devices and/or from the performance parameters of the available networks [3]. Also, it is important that this network changes process should be transparent for the user.

Based on the recent advances in the mobile phones, nowadays these devices could establish connections to multiple networks in a simultaneously way, which is referred to as multihoming [4]. This characteristic facilitates a seamless VHO process while it is seamless to the user [5] and allows the simultaneous transmission of multiple services across multiple network interfaces [6]. Several research projects use the multihoming strategy over a heterogeneous environment in order to achieve a load balancing [4]; [7-9] or to make a better distribution of the bandwidth charge [10,11]. In other studies this strategy was used as a decision tool for the VHO process [12-15].

Considering the aforementioned problems, we addressed our study about the Always Best Connected $(\mathrm{ABC})$ problem in heterogeneous wireless networks (HWN) in [16]. In this work we designed a proactive Vertical Handover Decision Algorithm (VHO-DA) based on user preferences, QoS requirements, and network conditions. Later in [17], we presents a load balancing optimization scheme; this scheme is composed by one mathematical model and a two-step algorithm based on the anchor-adjustment heuristic. In this paper, we also address the problem of load balancing across heterogeneous networks from the viewpoint of the operator. We present a multi-objective optimization model to solve the traffic load balancing problem into HWN using a multihoming strategy, and an evolutionary algorithm to solve it.

The remainder of this paper is structured as follows. In Section 2 we introduce the mathematical model that encodes the multi-objective function in order to obtain a global load balancing among HWN. In section 3 the load balancing algorithm based on strength pareto evolutionary algorithm is presented. The experimental results about the performance of our proposal are shown in Section 4. Finally, concluding remarks and directions for further research are given in Section 5 .

\section{Load Balancing Mathematical Model}

As it was mentioned in [17], the load balancing is an important strategy used by the mobile operators in order to allocate, in a fair way, the available resources in a network. However, this strategy implies, in many cases, the reallocation of mobile devices; therefore, it is necessary to consider the cost of connecting services to the new networks and the energy consumption of the mobile device. Considering the above statements and the possibility of the simultaneous use of multiple network interfaces by each mobile device [6], in this section we proposed a multiobjective - multihoming mathematical model.

\subsection{Decision Variable}

By assuming that mobile devices are able to perform multihoming in the network, we define the decision variable $x$ as a binary variable that specify if the service $s$ of the mobile $k$ is connected to the network $j$ or not (See Figure 1).

The variable is represented as follows:

$$
x_{j}^{k, s}= \begin{cases}1 & \text { if the service is connected to the network } \\ 0 & \text { otherwise }\end{cases}
$$




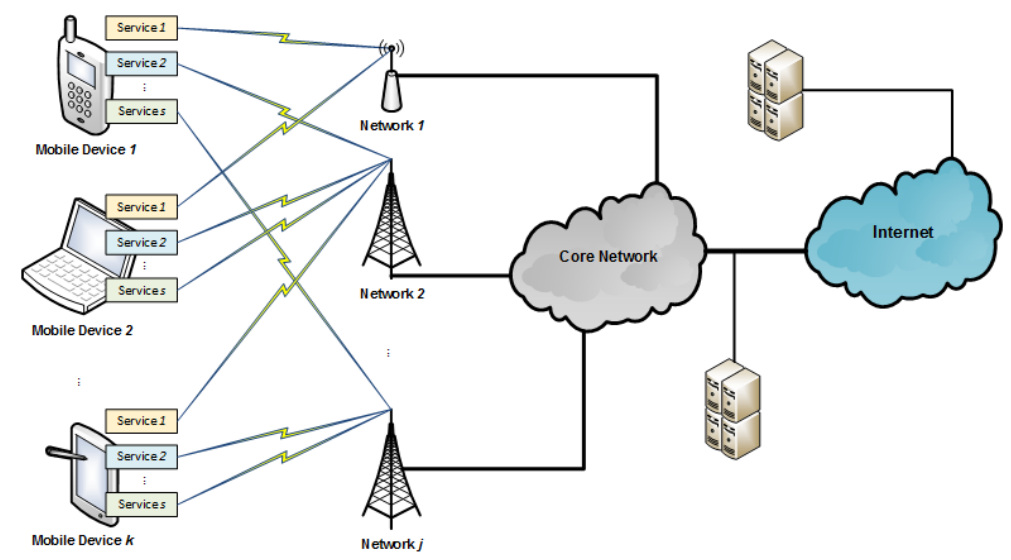

Figure 1: Multihoming Cellular System

\subsection{Objective Functions}

In order to design an efficient load balancing algorithm, the mathematical model is formulated under the premise of achieving an overall load balance in the wireless heterogeneous networks, whilst the connection cost and the energy consumption is also minimized. This function is expressed as: $\min (\alpha, \beta, \gamma)$, where $\alpha$ represents the load balancing function, $\beta$ the connection cost function, and $\gamma$ the energy consumption function.

\section{Load Balancing Function}

The load balancing function $(\alpha)$ is the main function of this model. This function determines the network traffic load by considering the demand of the services of the mobile devices in relation to the theoretical available bandwidth of the network. For this model, the load function is defined as: $\alpha=\max \left(\alpha_{j}\right), \forall j \in N$, where $j$ represents the destination network of the mobile device, $N$ the set of access networks and $\alpha_{j}$ the load of the network $j$.

We calculate $\alpha_{j}$ as the sum of demanded bandwidth $\left(D_{s}\right)$ of each connected service $(s)$, for each mobile $(k)$ over the theoretical available bandwidth of the network $\left(B W_{j}\right)$

$$
\alpha_{j}=\frac{\sum_{k} \sum_{s} D_{s} \cdot x_{j}^{k, s}}{B W_{j}}, \forall j
$$

\section{Connection Cost Function}

The function determines the maximum monetary cost of the mobile devices that are connected to the network. If a mobile device has at least one service connected to the network, its cost is taken into account to access the network. The connection cost function is expressed as: $\beta=\max \left(\beta_{j}\right), \forall j \in N$, where $j$ represents the network and $\beta_{j}$ the maximum cost of connected mobile devices to this network $\left(\right.$ Cost $\left._{j}\right)$.

$\beta_{j}$ is defined as:

$$
\beta_{j}=\sum_{k} \max _{s}\left(\operatorname{Cost}_{j} \cdot x_{j}^{k, s}\right), \forall j
$$




\section{Energy Consumption Function}

This function determines the energy consumption of the mobile devices that are connected to the network. If a mobile device has at least one service connected to the network, the consumption that generates for being connected to the network is taken into account in our model. We defined the energy function as: $\gamma=\max \left(\gamma_{j}\right), \forall j \in N$.

Whilst $\gamma_{j}$ is determined as the maximum consumption that is generated by mobile device $k$ for being connected to the $j_{t h}$ network

$$
\gamma_{j}=\sum_{k} \max _{s}\left\{\operatorname{Cons}\left(R S S_{k, j}\right) \cdot x_{j}^{k, s}\right\}, \forall j
$$

Finally, in order to guarantee consistency in our model, we consider that when a services $s$ is active in the device, it will generate a traffic demand $D_{s}$. Following the work presented in [18], the values of received signal strength are discretized at three levels: low, medium and high. Note that the power consumption is inversely proportional to the Received Signal Strength $(R S S)$ and therefore, a high signal level results in a low power consumption by the mobile radio interface through which communication is established.

Because multiple network interfaces can be used in a multihoming scenario, it is a privilege to be connected to those network which you receive better signals from, i.e. where less energy is consumed for being connected. Consumption levels derived from the RSS are modeled in the $\operatorname{Cons}\left(R S S_{k, j}\right)$ function.

$$
\operatorname{Cons}\left(R S S_{k, j}\right)= \begin{cases}1 & R S S_{k, j}>R S S_{t h 2} \\ 2 & R S S_{t h 1} \leq R S S_{k, j}<R S S_{t h 2} \\ 3 & 0<R S S_{k, j}<R S S_{t h 1} \\ 0 & R S S_{k, j}=0\end{cases}
$$

\subsection{Model Constraints}

Through the model constraints, we intend to guarantee the adjustment of this model to the real-life Telco networks. In this case, the model only allows the service connection to those networks that are in the coverage area, comply with the cost the user can assume to connect to the network, and offer enough bandwidth to meet the demand of service and an adequate power consumption according to the level of battery charge that the mobile device has.

\section{Demand Constraint}

The demand constraint states that the service can be only connected to a network that has enough bandwidth to meet its demand.

$$
D_{s} \cdot x_{j}^{k, s} \leq B W_{j}, \forall j
$$

\section{Cost Constraint}

The cost constraint states that the overall cost to access network $j\left(C_{o s t}\right)$, i.e. the cost to connect any service to network $j$ must be less or equal to the cost incurred by the user in the contract of the mobile device $k\left(\right.$ Cost $\left._{k}\right)$.

$$
\operatorname{Cost}_{j} \cdot x_{j}^{k, s} \leq \operatorname{Cost}_{k}, \forall k
$$




\section{Access Constraint}

Through the access constraint it is ensured that each service $s$ that is active on the mobile device $k$ is connected to a $N$ network and can only be connected to one network.

$$
\sum_{j} x_{j}^{k, s}=y_{k, s}, \forall k, \forall s
$$

\section{Reach Constraint}

This constraint ensures that only networks that exceed the defined signal strength threshold $\left(R S S_{t h}\right)$ are considered in the model assessment.

$$
R S S_{k, j} \geq R S S_{t h}, \forall j, \forall k
$$

\section{Power Consumption Constraint}

The power consumption constraint ensures that services only can be connected to those networks that offer lower power consumption, according to the current battery level of the mobile device.

$$
\operatorname{Cons}\left(R S S_{k, j}\right) \cdot x_{j}^{k, s} \leq \operatorname{Bat}_{k}, \forall j, \forall k
$$

where $\mathrm{Bat}_{k}$ is defined as:

$$
\text { Bat }_{k}= \begin{cases}1 & \text { Charge }_{k}<\text { Bat }_{t h 1} \\ 2 & \text { Bat }_{t h 2} \leq \text { Charge }_{k} \leq \text { Bat }_{t h 1} \\ 3 & \text { Charge }_{k}>\text { Bat }_{t h 2}\end{cases}
$$

A service $s$ of a mobile device $k$ is considered active when the constraints are satisfied at least for one of the networks; i.e. if the service can be connected to at least one of the networks available for the device. When the service $s$ is active, it consumes the traffic demand in the network that it is connected.

\section{Multi-Objective Evolutionary Algorithm}

In order to solve the multi-objective model, several strategies can be used. One of them is to evaluate each objective function in the model separately (mono-objective approach). The weight sum method is one of these strategies. However, it has several disadvantages, including not finding all optimal solutions if the solution set is not convex, and the need to normalize the functions so that no one predominates over the others [7].

For this reason we propose the use of an Multi-Objective Evolutionary Algorithm (MOEA) to find the best set of solutions for all objective functions at the same time. The chosen algorithm is the elitist type evolutionary algorithm SPEA (Strength Pareto Evolutionary Algorithm) proposed by Zitzler and Thiele [7,19]. The time complexity of this algorithm is upper bounded by $O\left(N M^{2}\right)$ in each generation, where $M$ is the population size and $N$ is the number of objectives. 


\section{Algorithm 1: SPEA Algorithm PSEUdo-CODE}

1: Generate a random population $M$

2: while not max number of generation do

3: Evaluate population according objective function

4: Calculate the fitness of each of the individuals

5: Classification Population based on fitness $(M, M \prime)$

6: $\quad$ Generate New Population $M_{t+1}$

7: Apply Binary Tournament Selection

8: Apply Crossover Operator

9: $\quad$ Mutation Operator

10: end while

11: Find Pareto Optimal Set.

\subsection{Chromosome Representation}

As starting point for implementing the evolutionary algorithm it is necessary to define the chromosome, i.e. the data representation of the solutions in the model. In the proposed mathematical model, the solutions can be expressed in a matrix representation (See Fig. 3). The rows represent mobile devices and the columns represent services; whilst the cell values represent the network in which the service will be connected, the value of zero is given when the service is not active and it is not connected to any network.

\begin{tabular}{|c|c|c|c|c|c|c|c|}
\hline $\begin{array}{c}\text { Node } \\
\text { s }\end{array}$ & $\begin{array}{c}\text { Node } \\
\mathbf{i}_{1}\end{array}$ & \multicolumn{3}{|c|}{... } & $\begin{array}{c}\text { Node } \\
1\end{array}$ & & \\
\hline $\begin{array}{c}\text { Node } \\
\text { s }\end{array}$ & $\begin{array}{c}\text { Node } \\
\mathbf{i}_{1}\end{array}$ & $\begin{array}{c}\text { Node } \\
\mathbf{i}_{2}\end{array}$ & $\begin{array}{c}\text { Node } \\
\mathbf{i}_{3}\end{array}$ & \multicolumn{3}{|c|}{$\cdots$} & $\begin{array}{c}\text { Node } \\
2\end{array}$ \\
\hline $\begin{array}{c}\text { Node } \\
\text { s }\end{array}$ & $\begin{array}{c}\text { Node } \\
\mathbf{i}_{1}\end{array}$ & $\begin{array}{c}\text { Node } \\
\mathbf{i}_{2}\end{array}$ & \multicolumn{3}{|c|}{$\cdots$} & $\begin{array}{c}\text { Node } \\
\mathbf{3}\end{array}$ & \\
\hline $\begin{array}{c}\text { Node } \\
\text { s }\end{array}$ & $\begin{array}{c}\text { Node } \\
\mathbf{i}_{1}\end{array}$ & $\begin{array}{c}\text { Node } \\
\mathbf{i}_{2}\end{array}$ & $\begin{array}{c}\text { Node } \\
4\end{array}$ & & & & \\
\hline
\end{tabular}

$\cdots \quad \cdots \quad \cdots$

\begin{tabular}{|c|c|c|c|c|c|}
$\begin{array}{c}\text { Node } \\
\mathbf{s}\end{array}$ & $\begin{array}{c}\text { Node } \\
\mathbf{i}_{1}\end{array}$ & $\begin{array}{c}\text { Node } \\
\mathbf{i}_{2}\end{array}$ & $\begin{array}{c}\text { Node } \\
\mathbf{i}_{3}\end{array}$ & $\begin{array}{c}\text { Node } \\
\mathbf{n}-1\end{array}$ & \multicolumn{2}{|l}{} \\
\hline $\begin{array}{c}\text { Node } \\
\mathbf{s}\end{array}$ & $\begin{array}{c}\text { Node } \\
\mathbf{i}_{1}\end{array}$ & $\begin{array}{c}\text { Node } \\
\mathbf{i}_{2}\end{array}$ & $\ldots$ & $\begin{array}{c}\text { Node } \\
\mathbf{n}\end{array}$ \\
\hline
\end{tabular}

Figure 3: Chromosome representation of the solution

The decision variables of the mathematical model can be directly obtained from the chromosome, for example if service 2 of the mobile device 3 is connected to network 4 , it means that $x_{4}^{3,2}=1$ and for the remaining $j$ networks the variable is 0 .

\subsection{Genetic Operators}

The crossover function takes two initial solutions (i.e. chromosomes), called parent solutions, and then created a new one from them. We define a crossover function based on the well-known 

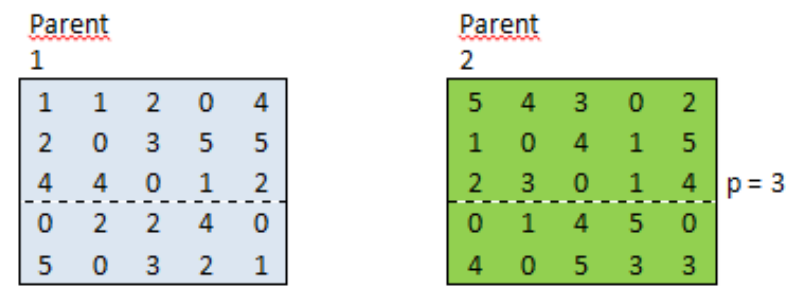

Child 1
\begin{tabular}{|lllll|}
\hline 1 & 1 & 2 & 0 & 4 \\
2 & 0 & 3 & 5 & 5 \\
4 & 4 & 0 & 1 & 2 \\
0 & 1 & 4 & 5 & 0 \\
4 & 0 & 5 & 3 & 3 \\
\hline
\end{tabular}

Child 2
\begin{tabular}{|lllll|}
\hline 5 & 4 & 3 & 0 & 2 \\
1 & 0 & 4 & 1 & 5 \\
2 & 3 & 0 & 1 & 4 \\
0 & 2 & 2 & 4 & 0 \\
5 & 0 & 3 & 2 & 1 \\
\hline
\end{tabular}

(a) Crossover function

Initial chromosome
\begin{tabular}{|lllll|}
\hline 1 & 1 & 2 & 0 & 4 \\
2 & 0 & 3 & 5 & 5 \\
4 & 4 & 0 & 1 & 2 \\
0 & 1 & 4 & 5 & 0 \\
4 & 2 & 5 & 3 & 3 \\
\hline
\end{tabular}

Final chromosome
\begin{tabular}{|lllll|}
\hline 1 & 1 & 2 & 0 & 4 \\
2 & 0 & 4 & 5 & 5 \\
4 & 4 & 0 & 1 & 2 \\
0 & 1 & 4 & 5 & 0 \\
4 & 2 & 5 & 3 & 3 \\
\hline
\end{tabular}

(b) Mutation function

Figure 4: Genetic Operators

single-point strategy proposed in [19]. The new solutions are generated by combining the first $p$ rows of the first parent solution with the last $k-p$ rows of parent second parent solution, and vice-versa. The Figure 4(a) shows an example of this operator. Since the solution of each mobile device in the parent chromosomes meets the constraints of the model, the solutions of the mobile devices that are part of the child solutions also meet the constraints since each mobile device represented in a row conserves its signal strength and battery level conditions as it moves from one chromosome to another. Also note that the inactive services are the same in the different solutions so they are conserved.

The mutation strategy is based on a random function that takes a mobile device service from its current to a new one as long as the constrains are met, i.e. the network will be connected to a feasible network. The function takes into account only the services that are activated and have more than one feasible network. Figure 4(b) shows an example of the mutation strategy. In this case, the service 3 of the mobile 2 is moved from network 3 to network 4

\subsection{Generation of Scenario Parameters}

Once the genetic operators are defined, the algorithm starts to generate pseudo-randomly parameters for each mobile. This parameters are the maximum cost that the user can assume to connect to one network $\left(\right.$ Cost $\left._{k}\right)$, the percentage values and battery charge levels $\left(\right.$ Bat $_{k}$ and Charge $\left._{k}\right)$, the signal strength values that each mobile perceives to each network $j\left(R S S_{k, j}\right)$, with its corresponding power consumption level parameter $\left(\right.$ Cons $\left._{k, j}\right)$.

When the scenario is created, we proceed to validate all constraints of model for each available network: demand, cost, reach and power consumption. After this validation, we store in a vector the networks that can be used to connect each service of each mobile device or the zero value ([0]) 
if the service cannot be connected to any network. The result is a feasibility matrix $\left(F_{a c t} t_{k, s}\right)$, this matrix is used to computed a matrix of active services $y_{k, s}$, where it is defined the probability of use for a mobile service, for each service that can be connected to at least one network. Once the active services are defined, a population of $M$ initial solutions and an $M$ ' elite (or external) population are randomly generated from both the feasibility and active services matrix. In our implementation, the value of $M$ and $M \prime$ are set to 20 and 4, respectively.

\section{Experimental Results}

In order to verify the correct operation of the proposed algorithm, we define four different experimental scenarios. For each one of them, we execute 500 iteration of the algorithm with different number of networks, mobile devices and services.

\subsection{First Experimental Scenario}

The aim of this scenario is to compare the quality of the solution obtained by our algorithm with respect to the optimal solutions obtained by solving each function separately. The optimal solutions were computed using GAMS system [20]. For this purpose, the scenario is composed by five mobile devices that will connect three services in three different available access networks. Tables 1 and 2 describe the parameters of bandwidth, cost, distribution of active services and network coverage for each mobile device.

Table 1: Network parameters

\begin{tabular}{|c|c|c|}
\hline Network Technology & Theoretical Bandwidth (Mbps) & Cost $_{j}$ (monetary units) \\
\hline LTE & 70 & 80 \\
WiFi g & 54 & 0 \\
HSPA + & 15 & 40 \\
\hline
\end{tabular}

Table 2: Bandwidth demand parameters

\begin{tabular}{|c|c|c|c|}
\hline Service & Voice & Video & Web \\
\hline Demand (Mbps) & 0.1 & 3 & 0.5 \\
\hline
\end{tabular}

To solve a multi-objective mathematical model through a general optimization software such as GAMS [20], it is necessary to convert the optimization problem into a single objective one. In this case, each objective function was optimized separately obtaining the solutions shown in Table 3. On the other hand the proposed algorithm converges rapidly to a set of 4 optimal solutions; three of them are unique. The results are presented in Table 4. Based on the feasible solutions obtained, we plot three different graphics in order to identify the Pareto-Optimal Front. These graphics are: load $(\alpha)$ vs. cost $(\beta)$, cost $(\beta)$ vs. consumption $(\gamma)$, and load $(\alpha)$ vs. consumption $(\gamma)$, as you can see in Figure 4:(a). In the load $(\alpha)$ vs. cost $(\beta)$ graph, the optimal Pareto front can be seen, because as the load decreases the cost grows. The cost $(\beta)$ vs. consumption $(\gamma)$ graph shows that solutions move in cost values of 80,120 and 160, and zero-cost solution is quite atypical. Finally, in the load $(\alpha)$ vs. consumption $(\gamma)$ graph can be seen that some optimal solutions are on value 4 and others on power consumption value 7 .

Making a comparison between the two sets of solutions obtained (Table 3 and 4), the proposed algorithm found several intermediate solutions belonging to the Pareto optimal front. These solutions cannot be found under mono-objective approaches because they are clearly not a linear 
Table 3: Mathematical model solutions obtained using GAMS

\begin{tabular}{|l|c|c|c|c|c|c|}
\hline & LTE (load) & WiFi g (load) & HSPA + (load) & $\alpha$ & $\beta$ & $\gamma$ \\
\hline Solution 1 & 0.086 & 0.080 & 0.080 & 0.086 & 160 & 4 \\
Solution 2 & 0 & 0.213 & 0 & 0.213 & 0 & 7 \\
Solution 3 & 0.103 & 0.080 & 0 & 0.103 & 160 & 4 \\
\hline
\end{tabular}

Table 4: Solution results for the first study case

\begin{tabular}{|l|c|c|c|c|c|c|}
\hline & LTE (load) & WiFi g (load) & HSPA + (load) & $\alpha$ & $\beta$ & $\gamma$ \\
\hline Solution 1 & 0.051 & 0.115 & 0.113 & 0.115 & 120 & 7 \\
Solution 2 & 0.051 & 0.124 & 0.080 & 0.124 & 80 & 4 \\
Solution 3 & 0.086 & 0.080 & 0.080 & 0.086 & 160 & 4 \\
\hline
\end{tabular}

combination of the objective functions. By looking at the solutions obtained by GAMS, it is possible to see that the solution 3 is dominated by solution 1, so that the solution 3 is not part of the Pareto optimal front. Moreover, it is highly improbable that the zero solution cost (found with GAMS) can be found by our algorithm, because this solution must connect all the services to the same network that goes against the main objective of our proposal.

Finally, in order to evaluate the quality of the solutions found by the algorithm, a performance metric called spacing was calculated. This metric, as its name implies, analyze the distribution of the solutions in a Pareto Front.

$$
S=\sqrt{\frac{1}{|Q|} \cdot \sum_{i=1}^{|Q|}\left(d_{i}-\bar{d}\right)^{2}}
$$

where:

$$
\begin{gathered}
d_{i}=\min _{k \in Q \wedge k \wedge \neq i} \sum_{m=1}^{M}\left|f_{m}^{i}-f_{m}^{k}\right| \\
\bar{d}=\sum_{i=1}^{|Q|} \frac{d_{i}}{|Q|}
\end{gathered}
$$

It is important to note that $d_{i}$ are the distance measure, $\bar{d}$ is the mean value of the above distance measure, and $f_{m}^{k}$ is the $m_{t h}$ objective function value of the $k_{t h}$ member of the population [7].

For our implementation the value of $S$ obtained was 21.505. This value was influenced in a strongest way by the difference in the cost values.

\subsection{Second Experimental Scenario}

We proceed to perform of the algorithm with the same external and elite population size $M=20, M \prime=4$; but changing to 20 mobile devices, 5 services for each device, and 7 access networks. This scenario was randomly generated according to the characteristics presented in Table 5 and 6 . The feasible solutions obtained are presented in Table 7 .

The solutions obtained show that the services present a tendency to be allocated into the networks with the highest capacity restricted only by the cost that each mobile could pay. The 
Table 5: Network parameters

\begin{tabular}{|c|c|c|c|c|c|c|c|}
\hline Network Technology & LTE & WiFi n & WiFi g & WiMAX & HSPA + & HSDPA & UMTS \\
\hline Theoretical Bandwidth (Mbps) & 70 & 300 & 54 & 15 & 15 & 2 & 0.3 \\
Cost $_{j}$ (monetary units) & 80 & 0 & 0 & 60 & 40 & 20 & 10 \\
\hline
\end{tabular}

Table 6: Bandwidth demand of services for second study case

\begin{tabular}{|c|c|c|c|c|c|}
\hline Service & Voice & Video & Web & Game & Chat \\
\hline Demand (Mbps) & 0.1 & 3 & 0.5 & 2 & 0.2 \\
\hline
\end{tabular}

Table 7: Network parameters

\begin{tabular}{|l|c|c|c|c|c|c|c|c|c|c|}
\hline & LTE & WiFi n & WiFi g & WiMAX & HSPA+ & HSDPA & UMTS & $\alpha$ & $\beta$ & $\gamma$ \\
\hline Solution 1 & 0.057 & 0.205 & 0.652 & 0.527 & 0.447 & 0.4 & 0.667 & 0.667 & 160 & 29 \\
Solution 2 & 0.086 & 0.205 & 0.585 & 0.587 & 0.493 & 0.467 & 0.587 & 0.587 & 240 & 27 \\
\hline
\end{tabular}

solutions obtained by our algorithm show that if the model constrains are met, then the algorithm trends to allocate the mobile services in those networks with the highest capacity and low cost

In addition, we plot three same graphics as the previous scenario (see Figure 5:(a)). In the first two graphs it is observed the optimal Pareto fronts. These first two graphs shows that in order to achieve a better load distribution across networks and to reach a lower power consumption of mobile devices, the services should be grouped into the higher-cost networks. However, it can be said that the load variations are little in comparison with the cost difference that can be obtained; so that the operator may prefer the solution which means lower cost. The value of the spacing metric $(S)$ was 35.542 , despite having three repeated solutions. The difference in the values of cost and load shows that the solutions are not equally distant.

\subsection{Third Experimental Scenario}

For this scenario, we only change the number of mobile devices to 500 in comparison with the latest scenario. The solutions obtained are shown in Table 8. Based on the achieved solutions for this scenario we can assert that our algorithm present a good grade of sensitivity. We can observe that small changes in the main parameters are reflected in the services allocation, which mean changes in the set of optimal solutions.

In Figure 6:(a) you can see, well defined, the optimal Pareto fronts in the first two graphs. This scenario also shows how small changes in load balancing produce appreciable changes in the cost function. The value of $S$ for this scenario was 30.168, again due to the wide differences in cost values.

\subsection{Fourth Experimental Scenario}

In this last scenario we increased the number of mobile devices to 10000 in order to validate the scalability of the algorithm. The other scenario parameters are kept as the scenario 2 . The feasible solutions are shown in Table 9.

Figure 6:(b) shows the optimal solutions found by our algorithm. It is possible to see that solutions trend to maintain the the same power consumption in both graphs, cost $(\beta)$ vs. consumption $(\gamma)$ and load $(\alpha)$ vs. consumption $(\gamma)$. The $S$ metric value was 1.0 , so that the solutions are fairly well spaced, being an unique solutions. The algorithm is fully adaptable to any situation within the proposed mathematical model designed. 

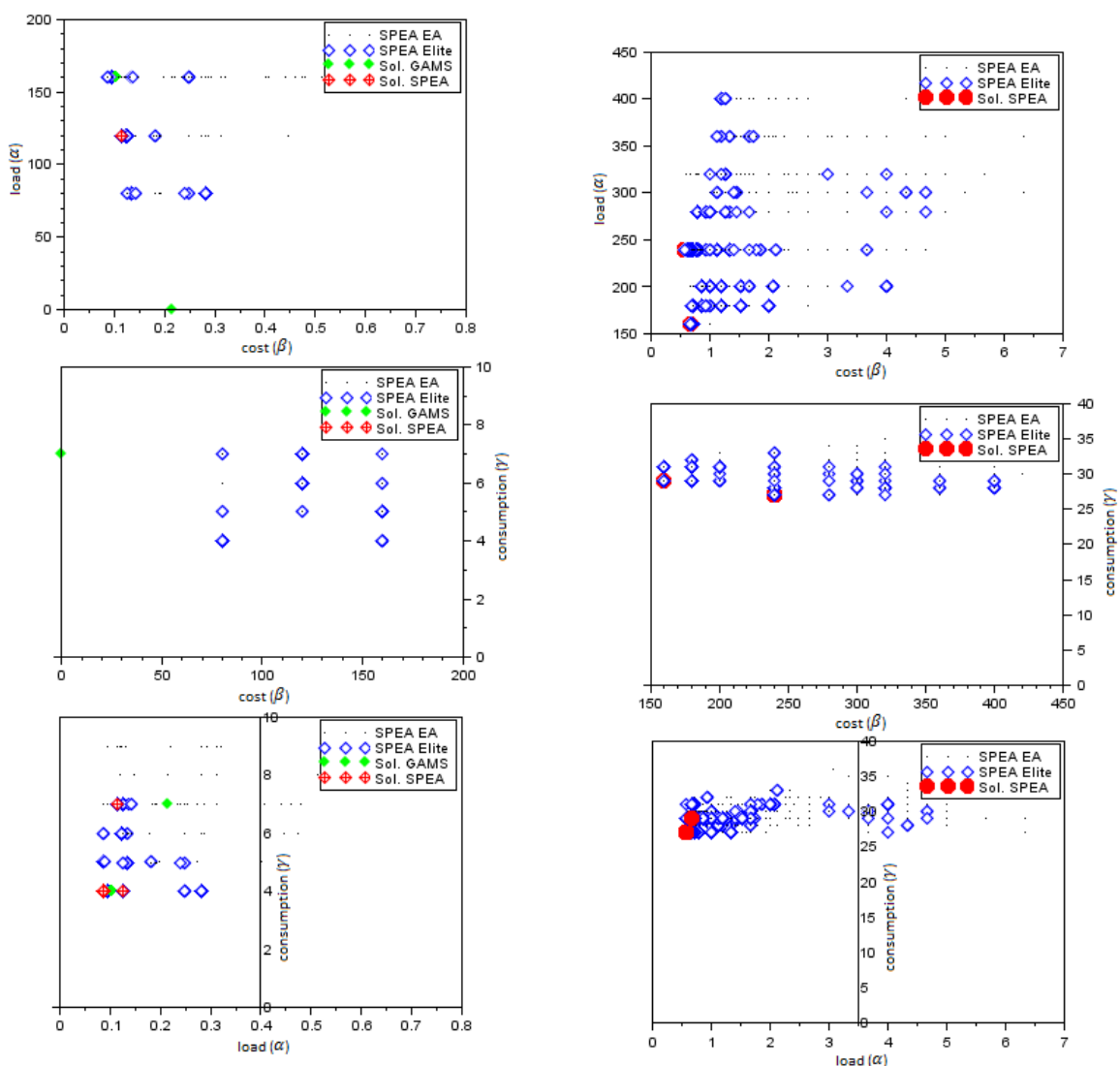

(a) First Study Case

(b) Second Study Case

Figure 5: 2D perspectives of solution distribution

Table 8: Solution results for the third study case

\begin{tabular}{|c|c|c|c|c|}
\hline & Solution 1 & Solution 2 & Solution 3 & Solution 4 \\
\hline LTE & 1.531 & 1.517 & 1.544 & 1.544 \\
WiFi n & 3.161 & 3.163 & 3.153 & 3.165 \\
WiFi g & 18.113 & 18.219 & 18.302 & 18.237 \\
WiMAX & 16.62 & 16.58 & 16.533 & 16.547 \\
HSPA+ & 27.247 & 26.947 & 26.747 & 26.747 \\
HSDPA & 48.6 & 48.467 & 48.6 & 48.533 \\
UMTS & 64.667 & 64.667 & 65.333 & 65 \\
\hline$\alpha$ & 64.667 & 64.667 & 65.333 & 65 \\
$\beta$ & 7560 & 7500 & 7440 & 7440 \\
$\gamma$ & 732 & 734 & 732 & 733 \\
\hline
\end{tabular}

After running the algorithm on this scenario, we prove that the algorithm maintains its characteristics of high sensitivity in the searching for optimal solutions regardless of the number of mobile devices on the problem. That means our algorithm is scalable. 
Table 9: Solution results for the fourth study case

\begin{tabular}{|c|c|c|c|c|}
\hline & Solution 1 & Solution 2 & Solution 3 & Solution 4 \\
\hline LTE & 30.46 & 30.26 & 30.263 & 30.463 \\
WiFi n & 65.56 & 65.63 & 65.62 & 65.57 \\
WiFi g & 358.61 & 358.64 & 358.61 & 358.55 \\
WiMAX & 329.43 & 328.86 & 328.69 & 329.6 \\
HSPA+ & 537.59 & 537.41 & 537.79 & 537.34 \\
HSDPA & 934.93 & 936.67 & 937.13 & 934.53 \\
UMTS & 1730 & 1730.33 & 1729 & 1731.33 \\
\hline$\alpha$ & 1730 & 1730.33 & 1729 & 1731.33 \\
$\beta$ & 152760 & 152520 & 152600 & 152680 \\
$\gamma$ & 15024 & 15027 & 15027 & 15022 \\
\hline
\end{tabular}
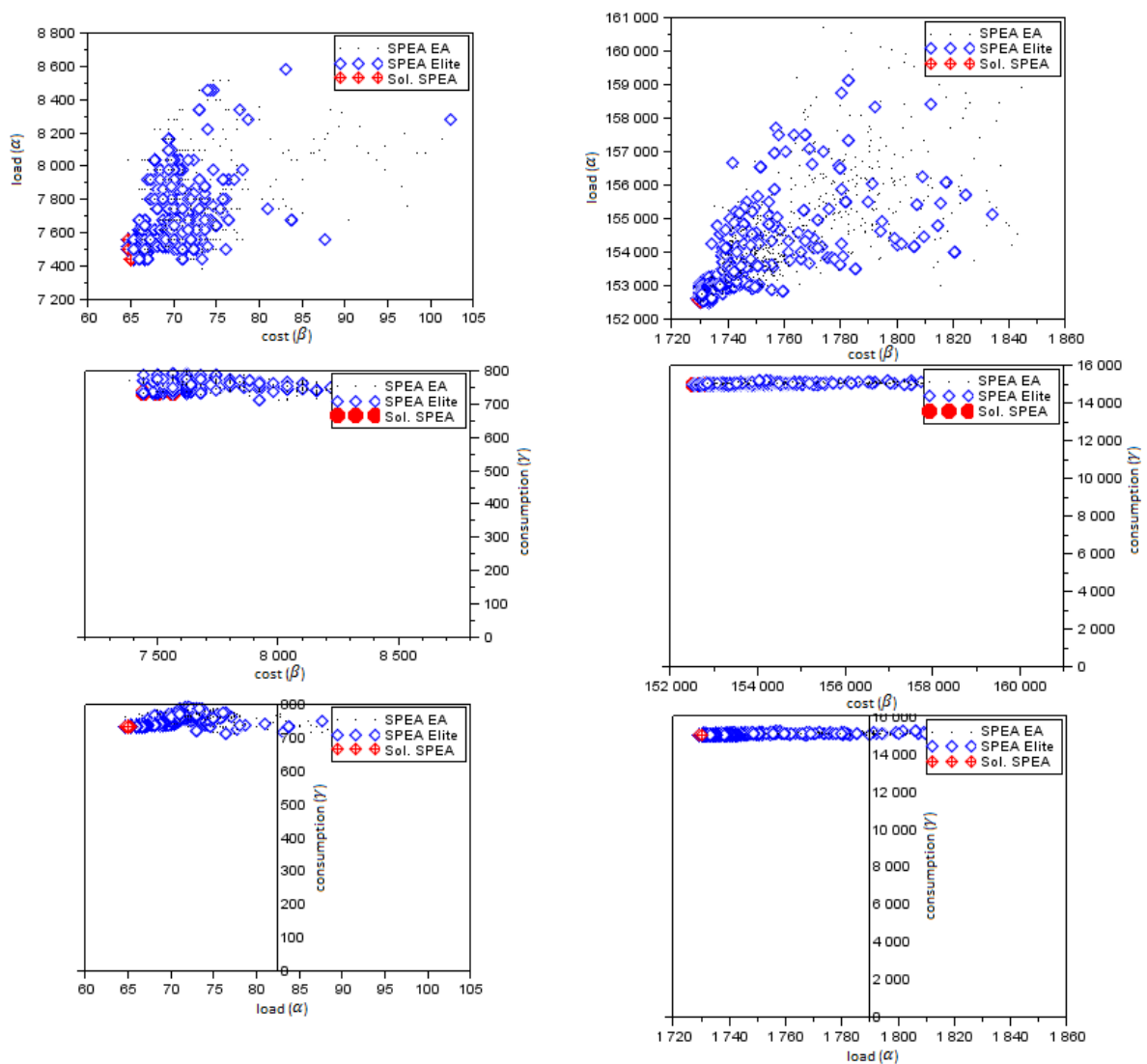

(a) First Study Case

(b) Fourth Study Case

Figure 6: 2D perspectives of solution distribution 


\section{Conclusions and Future Work}

We have presented a Multihoming Load Balancing Model in Heterogeneous Wireless Networks based on a multi-objective approach. In this model we take as objective functions the network load, connection cost, and energy consumption; with the aim of performing an efficient use of the capacity resources in the available networks.

Based on this model it was designed a Vertical Handover Algorithm (VHO) using evolutionary algorithms, specifically the Strength Pareto Evolutionary Algorithm (SPEA). Through the proposed environments we validate the correct operation of our algorithm; in the first scenario we validate the exactitude of the feasible solutions obtained by our algorithm in comparison to the solutions of the mathematical model using GAMS. In the other scenarios, we validate the sensibility and scalability of our evolutionary algorithm. The results obtained by our proposal were satisfactory and provided a starting point for the mobile network operator to run a VHO processes in their networks. With this process they could get an efficient use of their network resources, reduce the connection costs, and extend the battery life of mobile devices.

Due to we proposes a multi-objective optimization algorithm, the model is opens up to incorporate additional parameters as objective functions; these parameters could be obtained from both the available access networks and the mobile devices. As future work we propose to continue this research, we want to introduce the concept of fairness in the load balance optimization and also include the concept of quality of experience (QoE) in the objective functions.

\section{Acknowledgment}

The authors would like to thank to Administrative Department of Science, Technology and Innovation (COLCIENCIAS) for the financial support to Carlos Lozano-Garzon through the 528 - 2011 National Call for Doctoral Studies in Colombia

\section{Bibliography}

[1] IEEE Computer Society (2008); IEEE 802.21: Media Independent Handover Services, IEEE-SA Standards Board available at https://standards.ieee.org/getieee802/download/802.21-2008.pdf.

[2] Yan, X.; Şekercioğlu, Y. A.; Narayanan, S. (2010); An overview of vertical handover techniques: Algorithms, protocols and tools, Comput. Netw., ISSN 1389-1286, 54(11):1848-1863.

[3] Marquez-Barja, J.; Calafate, C.T.; Cano, J.C.; Manzoni, P. (2011); A Survey of Vertical Handover Decision Algorithms in Fourth Generation Heterogeneous Wireless Networks, Computer Communications, ISSN 0140-3664, 34(8):985-997.

[4] Sousa, B.M.; Pentikousis, K.; Curado, M. (2011); Multihoming Management for Future Networks, Mob. Netw. Appl., ISSN 1383-469X, 16(4):505-517.

[5] Paasch, C.; Detal, G.; Duchene, F.; Raiciu, C.; Bonaventure, O. (2012); Exploring Mobile/WiFi Handover with Multipath TCP, Proceedings of the 2012 ACM SIGCOMM Workshop on Cellular Networks: Operations, Challenges, and Future Design, New York, NY, USA: ACM, 31-36 (available at http://doi .acm.org/10.1145/2342468.2342476).

[6] Hyun-Dong, C.; Hyunjung, K.; Seung-Joon, S. (2013); Flow based 3G/WLAN vertical handover scheme using MIH model, 2013 International Conference on Information Networking (ICOIN), 658-663. 
[7] Donoso, Y.; Fabregat, R. (2007); Multi-Objective Optimization in Computer Networks Using Metaheuristics, Auerbach Publications, Boston, MA, USA.

[8] Capela, N.; Sargento, S. (2012); Optimizing network performance with multihoming and network coding, 2012 IEEE Globecom Workshops (GC Wkshps), pp. 210-215.

[9] Yang, R.; Chang, Y.; Sun, J.; Yang, D. (2012); Traffic Split Scheme Based on Common Radio Resource Management in an Integrated LTE and HSDPA Networks, 2012 IEEE Vehicular Technology Conference (VTC Fall), 1-5.

[10] Sungwook, K.; Varshney, P.K. (2002); An adaptive bandwidth reservation algorithm for QoS sensitive multimedia cellular networks, 2002 IEEE Vehicular Technology Conference (VTC Fall), 1475-1479.

[11] Sungwook, K.; Varshney, P.K. (2003); Adaptive load balancing with preemption for multimedia cellular networks, 2003 IEEE Wireless Communications and Networking (WCNC), 1680-1684.

[12] Li, M.; Fei, Y.; Leung, V.; Randhawa, T. (2003); A new method to support UMTS/WLAN vertical handover using SCTP, 2002 IEEE Vehicular Technology Conference (VTC Fall), 1788-1792.

[13] Bin, L.; Boukhatem, N.; Martins, P.; Bertin, P. (2010); Multihoming at layer-2 for interRAT handover, 2010 IEEE International Symposium on Personal Indoor and Mobile Radio Communications (PIMRC), 1173-1178.

[14] Eun, K.P.; Si, Y.H.; Hanlim, K.; Jong-Sam, J.; Seong-Choon, L.; Sang-Hong, L. (2008); Seamless Vertical Handover Using Multihomed Mobile Access Point, IEEE Global Telecommunications Conference, 2008 (IEEE GLOBECOM 2008), 1-4.

[15] Folstad, E.L.; Helvik, B.E. (2009); Managing availability in wireless inter domain access, International Conference on Ultra Modern Telecommunications Workshops, 2009. (ICUMT '09), pp. 1-6.

[16] Lozano-Garzon, C.; Ortiz-Gonzalez, N.; Donoso, Y. (2013); Mobile Network A Proactive VHD Algorithm in Heterogeneous Wireless Networks for Critical Services, International Journal of Computers, Communications $\&$ Control, ISSN 1841-9836, 8(3):425-431.

[17] Donoso, Y.; Lozano-Garzon, C.; Camelo, M.; Vila, P. (2014); A Fairness Load Balancing Algorithm in Heterogeneous Wireless Networks using a Multihoming Strategy, International Journal of Computers, Communications $\&$ Control, ISSN 1841-9836, 9(5):555-569.

[18] Mittal, R.; Kansal, A.; Chandra, R. (2012); Empowering Developers to Estimate App Energy Consumption, Proceedings of the 18th Annual International Conference on Mobile Computing and Networking, 317-328.

[19] Deb, K. (2001); Multi-Objective Optimization Using Evolutionary Algorithms, John Wiley \& Sons, Inc., New York, NY, USA.

[20] Rosenthal, R. E. (2015); GAMS a user's guide, GAMS Development Corporation., available at http://www.gams.com/dd/docs/bigdocs/GAMSUsersGuide.pdf. 